\title{
Nonlinear Sculpturing of Optical Spectra
}

\author{
Periklis Petropoulos* and Xin Yang \\ Optoelectronics Research Centre, University of Southampton, Southampton SO17 1BJ, UK \\ *Tel: (0044) 23805 92014, Fax: (0044) 23805 93142, e-mail: pp@orc.soton.ac.uk
}

\begin{abstract}
We present the experimental demonstration of a technique for the nonlinear generation of tailored broadband optical spectra. Our technique relies on the capability to precisely shape short optical pulses at the input of a highly nonlinear fibre. The precise pulse shape that needs to be fed to the nonlinear fibre is calculated from the spectral shape we wish to generate through inverse propagation calculations. A programmable optical filter is then used to transform the pulses of a short-pulse laser to the calculated waveform. An automated evolutionary algorithm is employed to monitor and optimise the filter characteristics. As an application example, we present the generation of a $10 \mathrm{GHz}$ ultra-flat (less than $1 \mathrm{~dB}$ power fluctuations), broadband (>30nm) optical spectrum, starting from a 2 ps mode-locked laser.
\end{abstract}

Keywords: Adaptive pulse shaping, evolution strategy, nonlinear fibre optics, optical communications.

\section{INTRODUCTION}

The capability to precisely manipulate the shapes of broadband optical spectra is central in several fields, ranging from communications to sensing and nonlinear optics. The nonlinear propagation of short optical pulses has often been used as a means for the generation of new optical frequencies. However, the relative strength of the various frequencies comprising the resultant broadened spectrum is largely determined by the characteristics of the nonlinear medium, and to address this, significant research effort has already been devoted to the development of optical fibres with suitably engineered nonlinear and dispersion properties. Rather than relying purely on the characteristics of the fibre, an alternative route towards obtaining a precisely shaped optical spectrum relies on applying spectral filtering after the nonlinear propagation [1]. Although this solution can be very effective, it is rather power-inefficient, since it is based on the removal of portions of power through filtering. Moreover, the bandwidth of the resultant spectrum is also restricted to that of the spectral filter itself.

In this paper, we outline our work on a novel approach for the generation of precisely shaped spectra based on adaptive shaping of a short pulse followed by propagation in a nonlinear medium. The particular application that we have considered is the generation of flat spectra that do away with the spectral ripples which are typical of the self-phase modulation (SPM)-induced spectral broadening of short optical pulses. As shown in Figure 1, in our approach, the pulse is required to take a form which after amplification and nonlinear propagation in an optical fibre will yield the required spectrum. Consequently, it is power efficient, since the signal is not filtered any further after it has been amplified, and has the potential to generate and manipulate light over a spectral range that greatly exceeds the operating bandwidth of the pulse shaping filter itself.

For a nonlinear element with given characteristics, the exact pulse waveform which yields the desired output spectrum after nonlinear propagation can be determined using backward propagation calculations based on the inverse split-step Fourier method (ISSFM) [2-4]. However, in order to overcome any experimental uncertainties relating to either of the characteristics of the pulse shaping filter, the nonlinear optical fibre or the nonlinear characteristics of the amplifier, we adaptively adjust the filtering function of the filter using an evolution strategy, by employing the measured optical spectrum at the system output as the feedback.

The following section describes the implementation of our technique through an experiment that has achieved the generation of a 12-nm spectrum exhibiting an 8-nm central region where the power fluctuations are smaller than $0.5 \mathrm{~dB}$. Then, by scaling the power at the input of the nonlinear fibre further, we generate a $35-\mathrm{nm}$ flat spectrum; these results are presented in Section 3.

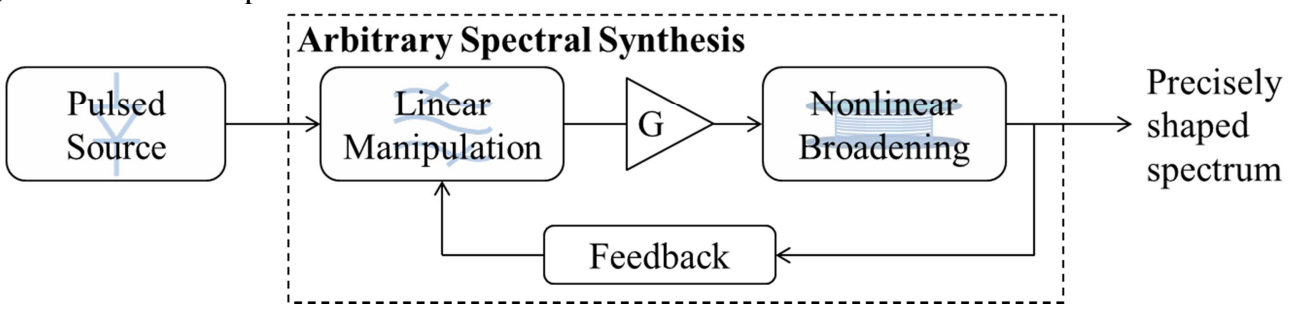

Figure 1 Principle of arbitrary nonlinear spectrum generation based on pulse pre-shaping

\section{NONLINEAR GENERATION OF A 12NM WIDE ULTRA-FLAT SPECTRUM}

Our experimental set-up is shown in Figure 2. The pulse source used in our experiments was a $10 \mathrm{GHz}$ modelocked laser (MLL) emitting nearly transform-limited Gaussian pulses with a full width at half-maximum of 2 ps. The pulses were shaped using a commercially available programmable phase and amplitude optical filter based 
on liquid crystal on silicon (LCOS) technology [5], which combined a high spectral resolution (7GHz) with acceptable insertion losses ( $\sim 5 \mathrm{~dB}$ ) (Finisar Waveshaper - W/S). The W/S had a dynamic range of 35dB, a polarization dependent loss of $0.2 \mathrm{~dB}$ and its individual spectral features could be programmed at $1-\mathrm{GHz}$ steps, much finer than the spectral line spacing of the $10 \mathrm{GHz}$ source itself. The setting resolution of the power attenuation and phase change of the W/S were $0.1 \mathrm{~dB}$ and $0.001 \mathrm{rad}$, respectively. In this work, the most promising feature of the W/S was the capability to dynamically change its filtering characteristics. The shaped pulses at the output of the W/S were amplified to $0.8 \mathrm{~W}$ and launched into a $1-\mathrm{km}$ long highly nonlinear fibre (HNLF) with a nonlinearity coefficient of $18 / \mathrm{W} / \mathrm{km}$, loss of $2.13 \mathrm{~dB} / \mathrm{km}$ and dispersion of $-1.7 \mathrm{ps} / \mathrm{nm} / \mathrm{km}$ at $1550 \mathrm{~nm}$. Taking into account the loss of the HNLF, the power at its output was $0.5 \mathrm{~W}$. The output of the HNLF was monitored by an optical spectrum analyser (OSA) and used as a feedback signal to adapt the pulse shaping function of the W/S, as will be described below.

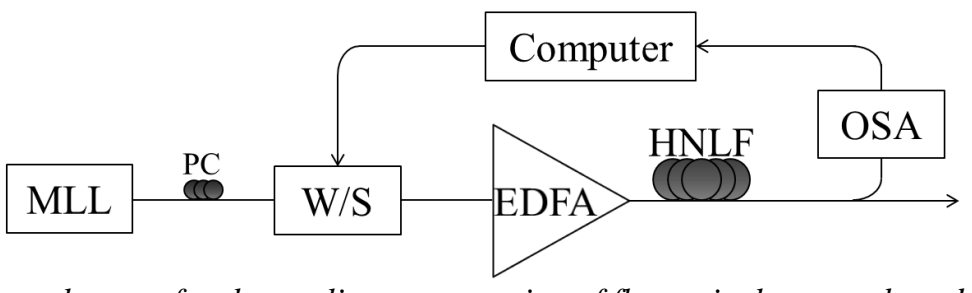

Figure 2 Experimental set-up for the nonlinear generation of flat optical spectra based on adaptive pulse shaping. PC: Polarisation Controller; EDFA: Erbium Doped Fibre Amplifier.

The generation process started with the definition of the target spectrum $U_{\text {target }}$, a third-order super-Gaussian spectrum in this case. The pulse waveform that was required at the input of the HNLF, and consequently the transfer function that needed to be fed to the W/S, was then determined using backward propagation calculations based on the ISSFM [4]. The calculations assumed that: (a) the exact characteristics of both the original pulses (in both phase and intensity) and the HNLF were known, (b) the amplifier prior to the HNLF was strictly linear, and (c) the phase and amplitude characteristics of the filter were accurately calibrated. However, it became clear even at early stages of experimentation that shaping had to be performed with such a high precision that these assumptions could not be tolerated in practice. This is evident in the result presented in Figure $3 \mathrm{a}$, which was obtained by simply programming the W/S with the transfer function calculated by the ISSFM. It was therefore deemed necessary to employ an adaptive pulse shaping mechanism aiming at minimizing the difference between the generated $U_{\text {meas }}$ and the target spectrum $U_{\text {target }}$ by dynamically adjusting the transfer function fed to the programmable spectral filter (the W/S). In order to achieve this, an evolutionary algorithm based on the covariance matrix adaptation evolution strategy (CMA-ES) was employed [6]. The algorithm used the misfit function $M F$ to quantify the difference between $U_{\text {meas }}$ and $U_{\text {target }}$, where

$$
M F^{2}=\int\left(U_{\text {meas }}-U_{\text {target }}\right)^{2} d \omega / \int U_{\text {meas }}^{2} d \omega .
$$

Since our goal was to improve the flatness of $U_{\text {meas }}$, evaluation of the $M F$ was restricted to include contribution from only wavelengths lying within the $5 \%$ bandwidth of $U_{\text {target }}$. In addition, we chose to act only on the phase profile of the pulse shaping function, since the power spectrum of the shaped signal could be accurately monitored using an OSA. The adjustment of the phase profile that was fed to the W/S was accomplished by adding a smaller perturbation represented by a weighted summation of first kind Chebyshev polynomials up to order $N$. The value of $N$ represents a trade-off between the accuracy of the optimization process and its efficiency. Here, the value of $N$ was chosen to be 20 . The use of Chebyshev polynomials has the advantage that when the weights are the same for all of the terms, their contribution to the entire summation is comparable. Consequently, instead of the phase profile itself, the 21 weights of Chebyshev polynomials were being optimized by the CMAES.

According to the principle of the CMA-ES, during the optimization process, in each generation, there were 13 offspring candidates generated by adding an array of weights which was Gaussian-distributed with zero mean over the parent candidate and repeating this procedure 13 times. Apart from the first generation, in which the parent candidate was an array of zero weights, the parent candidate was a weighted average of the best 6 out of 13 offspring candidates from the previous generation, with the offspring candidate with the smallest $M F$ value being rewarded with the largest weight. The variance of the Gaussian distribution was updated in a selfadaptation manner based on the concept of de-randomisation and cumulation [6]. The algorithm was terminated when the minimum $M F$ value in each generation converged to a sufficiently low value. The optimization process governed by the CMA-ES was carried out over a broad search space to ensure that the probability of finding the global minimum was sufficiently high.

Figure $3 \mathrm{~b}$ shows the generated spectrum when the adaptive system using CMA-ES was in place. The flatness of the generated spectrum was dramatically improved relative to the previous result of Figure 3a. A spectrum exhibiting a $3-\mathrm{dB}$ bandwidth of $12 \mathrm{~nm}$ with a central region of $8 \mathrm{~nm}$ where the fluctuation was less than $0.5 \mathrm{~dB}$ 

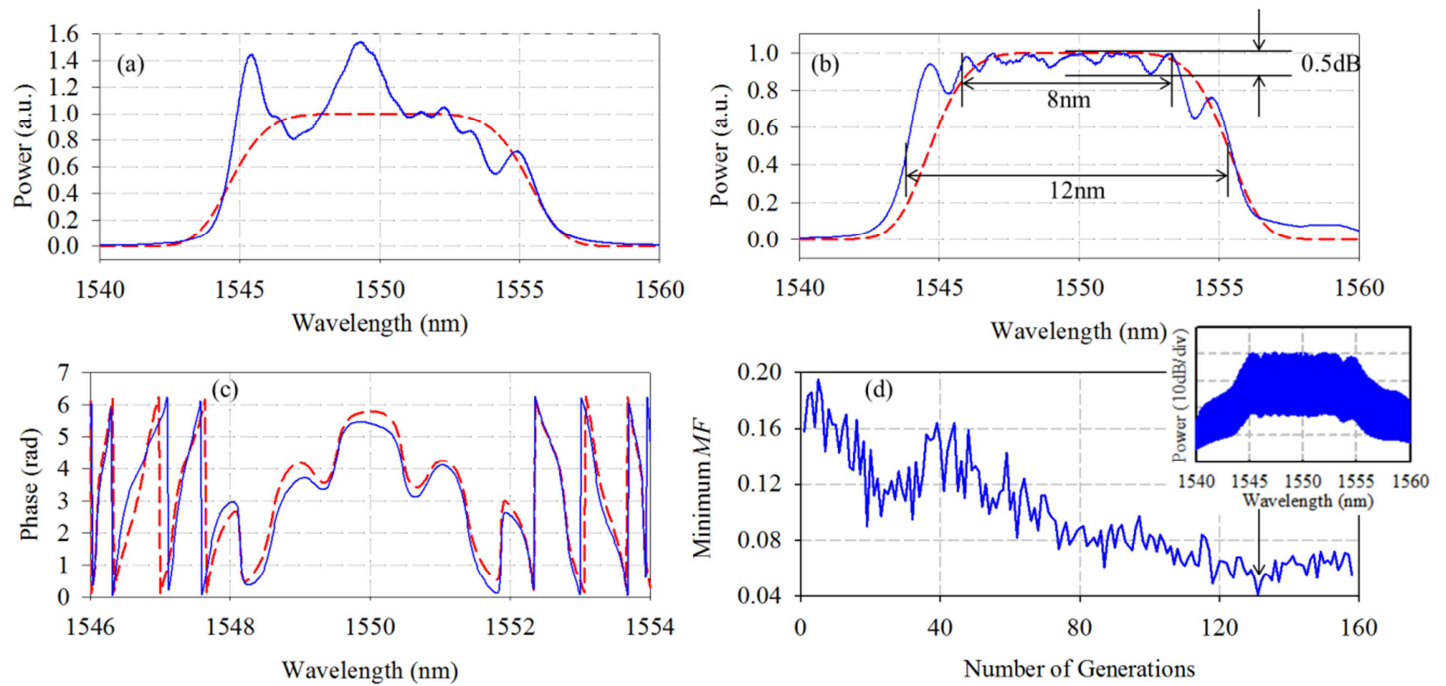

Figure 3 SPM spectra generated from (a) the waveform calculated directly from ISSFM and (b) after application of the adaptive system using CMA-ES in linear scale, respectively. Red dashed traces: target spectra, blue solid traces: measured spectra. Resolution bandwidth: 0.5nm. (c) Comparison between the phase profiles fed into the W/S before and after optimization. Red dashed trace: before optimization; blue solid trace: after optimization. (d) Evolution of Minimum MF value for each generation in CMA-ES. The inset shows high resolution (resolution bandwidth: $0.01 \mathrm{~nm}$ ) measurement results of the optimized spectrum with $M F=0.041$.

was nonlinearly generated. The inset to Figure $3 \mathrm{~d}$ shows a high resolution (resolution bandwidth $=0.01 \mathrm{~nm}$ ) measurement of this spectrum. The dense spectral lines accommodated in the spectrum can be observed. The uniform extinction ratio of the spectral lines across the entire bandwidth (limited by the resolution bandwidth of the OSA) is an indication of the good coherence properties of the generated signal. The phase profile of the W/S that gave rise to this spectrum is shown in the blue solid trace in Figure $3 \mathrm{c}$ and is compared to the numerically calculated one (red dashed trace). Even though the differences between the two traces were only relatively minor, the improvement in the flatness of the resultant spectrum was significant. This implies that the shaping of the spectral phase was very important for our application. Figure $3 \mathrm{~d}$ records the minimum $M F$ value in each generation. The $M F$ value achieved, which corresponds to the spectrum shown in Figure $3 \mathrm{~b}$ was 0.041 (for comparison the spectrum shown in Figure 3a exhibited a $M F$ value of 0.312). A five-fold improvement in the $M F$ value was observed within 130 generations. As there were 13 offspring candidates evaluated in each generation, the CMA-ES algorithm evaluated 1690 candidates in total. The large number of candidates considered made the algorithm robust, but also time-consuming. In the experiment, CMA-ES took approximately 2 hours to reach this solution.

\section{NONLINEAR GERERATION OF A 35NM WIDE ULTRA-FLAT FREQUENCY COMB}

Following the initial proof-of-principle experiments presented in the previous section, we progressed with a new experiment aiming at achieving a spectrum with a much broader bandwidth, and thereby proving that our technique has the potential to effectively shape spectra with bandwidths which are much broader than operating bandwidth of the pulse shaping filter. Since the parameter that we were mainly interested in optimising was the flatness at the top of the generated spectrum $U_{\text {meas }}$, the target spectrum $U_{\text {target }}$ was defined simply as a line of uniform spectral density across a $30 \mathrm{~nm}$ bandwidth centred at $1550 \mathrm{~nm}$. In addition, we did not use the ISSFM to calculate the pulse waveform leading to the target spectrum after nonlinear propagation. Instead, we relied on the capability of CMA-ES itself to find the optimum pulse shaping function, both in phase and amplitude.

The experimental set-up was the same as in the previous experiment (see Figure 2). The HNLF used in this experiment was $500 \mathrm{~m}$ long with a nonlinear coefficient of $19 / \mathrm{W} / \mathrm{km}$, loss of $2 \mathrm{~dB} / \mathrm{km}$ and a dispersion of $-0.87 \mathrm{ps} / \mathrm{nm} / \mathrm{km}$ at $1550 \mathrm{~nm}$. The output power of the EDFA before the HNLF was set to $2 \mathrm{~W}$ in this case, to stimulate sufficient nonlinearities for the spectral broadening. In the optimization process of CMA-ES, the shaping functions of both amplitude and phase were represented by a weighted summation of first kind Chebyshev polynomials of $10^{\text {th }}$ order. CMA-ES optimised these two weighted summations simultaneously. Consequently, in total, there were 22 elements in a vector of weights to be optimized. The settings of all the remaining parameters of CMA-ES were kept the same as in the previous experiment.

We first present in Figure 4a the experimentally generated spectrum at the output of the HNLF when no shaping had been applied. The spectrum exhibited a multitude of spectral peaks with large fluctuations. The best spectrum obtained after application of the adaptive shaping algorithm is presented in Figure $4 \mathrm{~b}$. The trace shows significant improvement in the spectral flatness. A spectrum with a 35-nm 3-dB bandwidth was achieved, 

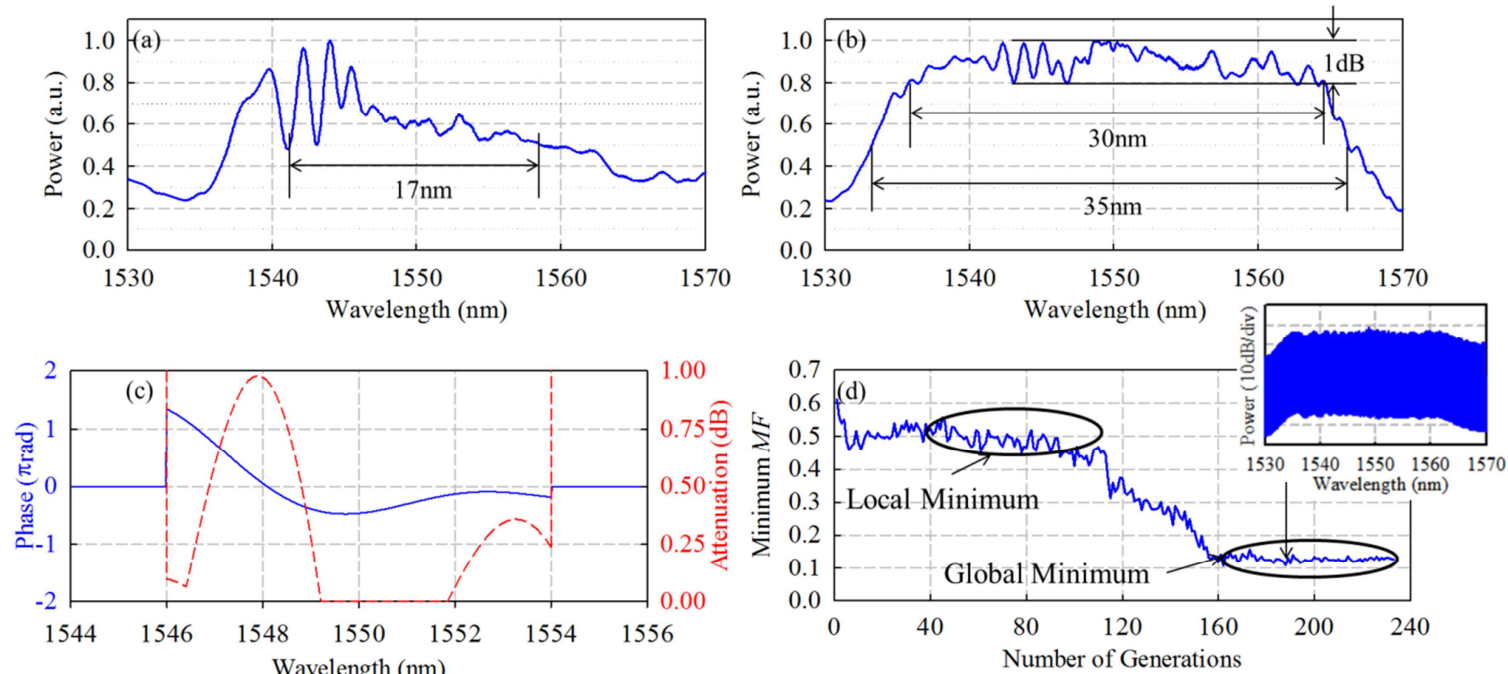

Figure 4 SPM spectra generated from (a) 2ps Gaussian pulses, and (b) after the application of the adaptive system using the CMA-ES in linear scale. Resolution bandwidth: 0.5nm. (c) Transfer function of the W/S after the optimization process based on CMA-ES. Solid blue trace: Attenuation profile; Dashed red trace: Phase profile. (d) Evolution of Minimum MF value for each generation in CMA-ES. The inset shows high resolution (resolution bandwidth: $0.01 \mathrm{~nm}$ ) measurement results of the optimized spectrum with $M F=0.12$.

exhibiting a uniform spectral density in a central region of $30 \mathrm{~nm}$ to within $1 \mathrm{~dB}$. A high resolution measurement (resolution bandwidth $=0.01 \mathrm{~nm}$ ) of the generated frequency comb is presented in the inset of Figure $4 \mathrm{~d}$. The generated ultra-flat frequency comb had the same line spacing as the pulse source. Therefore, approximately 375 spectral lines were accommodated within the $30 \mathrm{~nm}$ flat central region. Figure $4 \mathrm{c}$ shows the pulse shaping function we obtained after optimization. Note that the shaping bandwidth of the W/S was just $8 \mathrm{~nm}$ in our experiment, much narrower than the bandwidth of the spectrally flattened signal. Finally, Figure $4 \mathrm{~d}$ plots the minimum $M F$ value versus the number of generations - a five-fold improvement in the $M F$ value was achieved within 240 generations (3120 iterations). It is worth mentioning that the result of Figure $4 \mathrm{~d}$ acts as a typical example of the capability of CMA-ES to escape from a local minimum. The plot shows that the process had been trapped at a local minimum of around 0.5 for about 60 generations. Then, the self-adapted transition of the step size re-initiated a decrease in the $M F$ value. Finally, the adaptive algorithm converged to an optimum transfer function corresponding to a global minimum of around 0.12 .

\section{CONCUSION}

We presented a technique that allows tailoring of the spectra generated through nonlinear propagation in a power-efficient manner. The technique relies on pre-shaping the waveforms launched into a nonlinear medium (a nonlinear optical fibre in our case), such that the desired spectrum would be obtained at the output. Adaptive spectral broadening through adoption of an evolutionary algorithm proved essential in achieving the required accuracy during the shaping process. Our experiments aimed at generating ultra-flat frequency combs, originating from a telecommunications MLL. This technique provides highly controllable spectral broadening and, at the same time, the capability to power-efficiently harness optical spectra over a spectral range that greatly exceeds the operating bandwidth of the pulse shaper itself.

\section{REFERENCES}

[1] A.M. Clarke, et al., Reconfigurable Optical Pulse Generator Employing a Fourier-Domain Programmable Optical Processor. Journal of Lightwave Technology, 2010. 28(1): p. 97-103.

[2] S. Shuxian, et al., Numerically reversing the nonlinear wave propagation in single-mode optical fiber. IEEE Lasers and Electro-Optics Society 1999 Annual Meeting, 1999: paper WZ4.

[3] P.J. Almeida, et al., Generation of ultra-flat SPM-broadened spectra in a highly nonlinear fiber using pulse pre-shaping in a fiber Bragg grating. 2005 Optical Fiber Communications Conference: paper OThA4.

[4] X. Yang, et al., Pulse shaping-assisted nonlinear spectral broadening. 2011 Conference on Lasers \& Electro-Optics Europe \& 12th European Quantum Electronics Conference CLEO EUROPE/EQEC, 2011: paper CI2.1 TUE.

[5] G. Baxter, et al., Highly programmable Wavelength Selective Switch based on Liquid Crystal on Silicon switching elements. 2006 Optical Fiber Communication Conference, 2006: paper OTuF2.

[6] N. Hansen, A. Ostermeier, Completely derandomized self-adaptation in evolution strategies. Evolutionary Computation, 2001. 9(2): p. 159-195. 